\title{
MASSIVE STARS AND GIANT HII REGIONS: THE HIGH-ENERGY PICTURE
}

\author{
THIERRY MONTMERLE \\ Service d'Astrophysique, \\ Centre d'Etudes Nucléaires de Saclay \\ 91191 Gif-sur-Yvette Cedex, France
}

\begin{abstract}
Giant HII regions contain highly energetic objects: luminous, massive stars (including Wolf-Rayet stars) generating powerful winds, as well as, often, supernova remnants. These objects interact with the surrounding gas by creating shock waves. Part of the energy input is radiated away in the form of X-rays; also, protons and electrons may be accelerated in situ and generate $\gamma$-rays by collisions with the ionized gas. In addition, the stars themselves (including the accompanying low-mass PMS stars) are sources of X-rays, and W-R stars may emit continuum $\gamma$-rays and are associated with nuclear $\gamma$-ray lines seen in the interstellar medium. Therefore, both through the stars they contain and through interactions within the gas, giant HII regions are, in addition to their more traditional properties and over nearly 7 decades in energy, important sources of high-energy radiation.
\end{abstract}

\section{Introduction: which "high energies"?}

\subsection{WHY?}

"Giant HII regions" are gaseous regions excited by the ionizing flux of luminous, massive stars (0, early B, and Wolf-Rayet stars), which are subject to an intense mass-loss in the form of dense, fast winds (from $10^{-6} \sim 10^{-4} \mathrm{M}_{\odot}$, at up to $\sim 4000 \mathrm{~km} . \mathrm{s}^{-1}$; see Langer 1989, and this volume, for the latest developments). These winds in turn act on the surrounding material and create large cavities, bounded by a shock wave. Additional energy can be input by the explosion of type II supernovae, i.e., the endpoint of evolution of massive stars.

Through a variety of processes, which we will review in this paper, high-energy photons are emitted from the sub-keV range (soft X-rays) all the way to the GeV range $(\gamma$ rays). (For a broader perspective, see Montmerle 1987.) Also, high-energy particles are present: non-thermal radio emission reveals the presence of $\mathrm{MeV}-\mathrm{GeV}$ electrons (depending on the value of the magnetic field), and super-GeV protons may be accelerated in situ and interact with the ionized material. Although the topic will not be discussed here, it is worthy of note that antiprotons and neutrinos may also be produced as secondary particles after high-energy collisions in the ionized gas (see Montmerle 1988 for details).

\subsection{HOW ?}

Since the physical context of the high-energy phenomena is not discussed elsewhere in this volume, it is perhaps appropriate to briefly summarize here the physical processes relevant to giant HII regions, and their implications. 
a) X-rays:

The X-rays are thermal (bremsstrahlung of $a \approx 10^{7} \mathrm{~K}$ plasma) when they result from shocks (wind flows, wind bubbles, associated with 0 and $\mathrm{W}-\mathrm{R}$ stars), but also from strong flares occurring on low-mass pre-main sequence (PMS) stars. (We shall see below that, because they are very numerous and located in the same molecular clouds as giant HII regions, such stars can make a significant contribution to the overall X-ray budget.) These $\mathrm{X}$-rays are in general optically thin, hence give information throughout the whole emitting region.

But the X-rays may also be non-thermal, in which case the spectrum is a power-law. Compton scattering of the ambient radiation by high-energy electrons (themselves seen by means of their non-thermal radio emission) has been invoked in the case of the winds of W-R stars.

b) Continuum $\gamma$-rays:

For instrumental reasons, these $\gamma$-rays are known in the Galaxy only above $\sim 30 \mathrm{MeV}$ (the threshold for electron pair production in spark chamber detectors). They are produced by collisions of $\gtrsim \mathrm{GeV}$ cosmic rays (primarily $\mathrm{p}$ and $\alpha$ ) with interstellar matter (primarily $\mathrm{H}$ and $\mathrm{He}$ ): protons collisions give neutral pions which decay into $2 \gamma$-rays, as well as charged pions which decay into charged muons and subsequently in electrons and positrons. These secondary $\mathrm{e}^{+}$and $\mathrm{e}^{-}$, as well as primary electrons, also produce $\gamma$-rays in the same energy range by bremsstrahlung in the electric field of interstellar atoms. In addition to the standard galactic cosmic rays, protons and electrons can be accelerated locally by shock waves associated with stellar winds and SNRs.

c) $\gamma$-ray lines:

In addition to generating high-energy $\gamma$-rays as described above, collisions between lowenergy cosmic rays with interstellar nuclei, or stellar nuclear reaction products dispersed by SN explosions or stellar winds, may result in the emission of $\gamma$-ray lines. This is the case when nuclei in excited states or radioactive isotopes are produced. So far, however, the only positively detected $\gamma$-ray line in the Galaxy is the $1.8 \mathrm{MeV}$ line associated with the $\beta^{+}$decay of ${ }^{26} \mathrm{Al}$, a product of stellar nucleosynthesis. Since the corresponding half-life is short $\left(\sim 10^{6}\right.$ yrs), the presence of this line testifies to the existence of fresh nucleosynthesis in the interstellar medium.

\section{X-rays and $\gamma$-rays from stars}

\subsection{MASSIVE STARS}

The bulk of what we know today about the X-ray emission from stars has been obtained by the Einstein satellite. X-ray emitting stars have been discovered throughout the HR diagram (e.g., Rosner, Golub, and Vaiana 1985), the 0 stars spanning the luminosity range $\mathrm{L}_{\mathrm{X}} \sim 10^{31}-10^{34} \mathrm{erg} \cdot \mathrm{s}^{-1}$. But the 0 stars have a unique property: the ratio $\mathrm{L}_{\mathrm{X}} / \mathrm{L}_{\mathrm{bol}}$ of their X-ray luminosity to their bolometric luminosity is approximately constant, $\approx 10^{-7}$ (Chlebowski, Harnden, and Scortino 1989), and remarkably independent of main stellar parameters such as mass, radius, rotation velocity, effective temperature, although it may slightly depend on the environment (Chlebowski 1989). The reason for this is not understood. Proposed models for the X-ray emission involve thermal bremsstrahlung from shocks within the wind (see the discussion by S. Owocki, this volume), or nonthermal Compton radiation from the high-energy electrons seen in the radio range. 
In the case of W-R stars, the X-ray luminosities span a comparable range, from $10^{32}$ to $10^{34} \mathrm{erg} . \mathrm{s}^{-1}$. The average X-ray energy (or temperature, depending on whether the X-rays are thermal or non-thermal) is $\sim 1 \mathrm{keV}$, and the column density of interstellar gas is $\sim 10^{21}$. $10^{22} \mathrm{~cm}^{-2}$. In principle, the shape of the X-ray spectrum should indicate whether we are dealing with thermal (exponential in the case of bremsstrahlung) or non-thermal (powerlaw) processes.

In practice, however, for lack of spectral resolution, the answer is often ambiguous, and one has to resort to indirect arguments, drawn for instance from radio studies. Nonthermal radio emission is found to be common in the case of 0 stars (Bieging, Abbott, and Churchwell 1989); until recently, it was thought that, in the case of single W-R stars, the radio emission was thermal (free-free emission from the ionized wind; see, e.g., Hogg 1989, or Williams et al. 1990), but an increasingly large number of non-thermal cases are now being found (see the paper by M. Felli, this volume). This, in turn, reveals the presence of $\lesssim \mathrm{GeV}$ electrons (Lorentz factor $\gamma \lesssim 10^{3}$ ) in $\approx \mathrm{mG}$ magnetic fields, likely accelerated by shocks and/or turbulence in the wind itself. Further, since the wind is an efficient absorber of radio waves because it is ionized, the observed non-thermal radio flux can come only from the outer parts of the wind, i.e., $R>R\left(\tau_{\text {radio }}=1\right) \approx a$ few $100 R_{*}$, as shown by Pollock $(1987 \mathrm{a}, \mathrm{b})$. This has interesting implications: since the electron spectrum is a power law (number density of electrons $\propto \gamma^{-p}$, with $p \sim 3$ ), other parts of their spectrum contribute at other wavelengths: $\mathrm{keV}$ X-rays are produced by inverse Compton scattering by electrons with $\gamma \sim 10$ and leave the star provided that they are emitted at $R>R\left(\tau_{X}=1\right) \approx 100 \mathrm{R}_{*}$. By the same token, $>100 \mathrm{MeV} \gamma$-rays may be produced by bremsstrahlung of electrons with $\gamma \sim$ $10^{3}$ in the dense layers of the photosphere (where $\tau_{\gamma} \ll 1$ ). As a bonus, one may even explain the IR spectrum around $2 \mu \mathrm{m}$ by synchrotron emission of electrons with $\gamma \sim 10^{4}$ and $B \approx 1 \mathrm{G}$ also at the photospheric level (where $\tau_{2 \mu \mathrm{m}} \ll 1$ as well).

\subsection{LOW-MASS PMS STARS}

At the other end of the mass spectrum $\left(\mathrm{M} \sim 0.5-2 \mathrm{M}_{\odot}\right)$, PMS stars are also X-ray emitters, with luminosities $\mathrm{L}_{X} \sim 10^{31-32} \mathrm{erg} . \mathrm{s}^{-1}$. They are thus individually $10^{4-6}$ times more powerful in X-rays than their main sequence counterparts, and, in particular, the Sun (see, e.g., Montmerle et al. 1983, Feigelson 1987, Montmerle and André 1988). These stars are known under the generic term "T Tauri stars" (which include several sub-classes, see Bertout 1989). They are important in our context because they are much more numerous than massive stars while being also associated with molecular clouds. The X-rays are here produced by thermal bremsstrahlung of powerful flares having $\mathrm{kT} \sim 1 \mathrm{keV}$. The

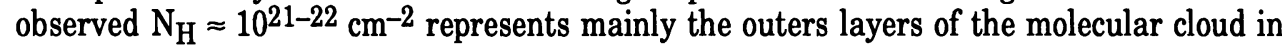
which they are embedded. This intense solar-like activity results from the fact that these stars are entirely convective or have deep outer convective zones (the depth depends on the age), which, together with rotation, generates a dynamo effect (e.g., Bouvier 1990).

\section{Interaction of massive stars with the surrounding medium}

We take here into account the fact that W-R stars generate a lot of mechanical energy $\left(\mathrm{L}_{\mathrm{W}} \approx\right.$ $10^{38} \mathrm{erg.s} \mathrm{s}^{-1}$ ) and that, being the "cousins" of 0 and B stars grouped in associations, they contribute in a major way to the creation of cavities in the giant HII regions excited by the 0 and early B stars.

The standard model describing the interaction between massive stars (wind + 
ionizing flux) and the surrounding medium is that of the "interstellar bubble" (Weaver et al. 1977). One feature of this model is that it predicts the existence of a thin HII shell around the cavity (i.e., $\mathrm{R}_{\text {shell }} \ll \mathrm{R}_{\text {cavity }}$ ); also, the shock is essentially adiabatic, and a large fraction $(\sim 20 \%)$ of the wind energy is imparted to the HII shell. However, there are many famous cases (for instance the Rosette nebula; see also LMC nebulae, e.g., Meaburn et al. 1989) in which the shell is thick $\left(R_{\text {shell }} \approx R_{\text {cavity }}\right)$. Also, the kinetic energy imparted to the HII shell is often much smaller than predicted by the standard model ( 1\%, see, e.g., Chu 1983, for the case of W-R nebulae).

The reason for the disagreement lies at least in part in the physical conditions prevailing near or at the shock. Indeed, the basic question is whether or not the shock is adiabatic: in the extreme case in which the shock is isothermal, the ratio of the pressures downstream and upstream of the shock can go to infinity, and all the wind kinetic energy can be radiated away, leaving nothing for the outer motion of the HII shell.

Recent works have suggested possible explanations. Dorland, Montmerle, and Doom (1986), and Dorland and Montmerle (1987) have explored the possibility that electron conduction may play a more important role than previously thought. Indeed, as shown in fusion plasmas ("Tokamaks"), electron conduction is non-linear in the case of steep temperature gradients, i.e. when the characteristic temperature length scale $\mathrm{L}_{\mathrm{T}} \lesssim 500 \lambda_{0}$, where $\mathrm{L}_{\mathrm{T}}=\mathrm{T}_{\mathrm{c}} / \Delta \mathrm{T}_{\mathrm{c}}, \Delta \mathrm{T}_{\mathrm{c}}$ being the temperature gradient obtained with classical conduction, and $\lambda_{0}$ is the electron mean free path, proportional to $\mathrm{v}^{4}$ (Luciani, Mora, and Pellat 1985; see also Campbell 1984). In that case, electron conduction at a given point becomes "non-local", i.e., depends on the (non-maxwellian) electron distribution function within $\sim$ a few m.f.p. of that point. The result is that the actual temperature gradient is governed by the electrons, giving rise to a softer temperature gradient than $\Delta \mathrm{T}_{\mathrm{c}}$. In nebulae like the Rosette nebula, one finds $\mathrm{L}_{\mathrm{T}} / \lambda_{0} \sim 150$ in the vicinity of the shock, which justifies a nonlinear treatment. Then the plasma downstream of the shock is made up of hot electrons and cold ions: X-ray emission results from bremsstrahlung of the former in the electric field of the latter, hence gives temperatures lower than for a standard downstream plasma: for instance, a $4000 \mathrm{~km} . \mathrm{s}^{-1}$ wind (typical of W-R stars) impinging on the surrounding HII region gives a temperature in the shock vicinity of $\approx 10 \mathrm{keV}$ instead of the $\approx 40 \mathrm{keV}$ computed in the standard way (i.e., $\mathrm{kT}=1 / 2 \mu \mathrm{mv}^{2}$, where $\mu$ is the mean molecular weight). The corresponding ratio $\mathrm{L}_{\mathrm{X}}(\mathrm{IPC}) / \mathrm{L}_{\mathrm{w}}$ of the diffuse X-ray luminosity in the Einstein IPC band over the wind power (in a $4 \pi$ cavity) is $\sim 2 \times 10^{-4}$ : for a single W-R star, this amounts to $\mathrm{L}_{\mathrm{X}, \mathrm{W}-\mathrm{R}} \approx 4 \times 10^{34}$ erg. $\mathrm{s}^{-1}$ (see Dorland and Montmerle 1987 for details).

In another approach, Breitschwerdt and $\operatorname{Kahn}(1988,1989)$ have shown that acoustic instabilities exist at the wind shock. The growth rate is short enough that turbulence sets in the vicinity of the shock: the characteristic time $t_{\text {wave }} \simeq t_{\text {sound }}$ (HII region) $\ll t_{\text {dyn }}$ (HII region). In turn, this leads to a rapid mixing of the hot stellar wind and the much cooler HII gas, such that the cooling rate grows by $\sim 1$ order of magnitude. Here again, a significant fraction of the wind energy is radiated away near the wind shock, even if, as it turns out, this instability is partly inhibited by magnetic fields. The evolution of the structure of the HII shell and the energy loss have yet to be calculated, but, given the shape of the cooling function (see e.g., Gaetz and Salpeter 1983), it is likely that the energy will be radiated at the peak of this curve, i.e., $\mathrm{T} \sim 10^{6} \mathrm{~K}$, in other words in the sub-keV domain. 


\section{X-rays from giant $\mathrm{HII}$ regions}

\subsection{STELLAR WIND SHOCKS}

Quantitative studies have been done by Dorland and Montmerle (1987) and Montmerle (1987) on the Orion, Rosette, and Carina nebulae. Since the last nebula is the only one of these which contains W-R stars, we will focus our attention on it. The available X-ray data are made up of Einstein observations (IPC images for $\sim \mathrm{keV} \mathrm{X-rays,} \mathrm{MPC} \mathrm{flux} \mathrm{for} \mathrm{X-rays}$ of a few keV; Seward and Chlebowski 1982, Chlebowski et al. 1984), and 0SO-8 flux for energies up to $20 \mathrm{keV}$ (Becker et al. 1976). More recent data were obtained up to $10 \mathrm{keV}$ by Tenma (Koyama et al. 1990). These last three experiments were of scintillating collimator type (i.e., not imaging), but their field of view included the whole nebula. Two components can clearly be distinguished, both fitted by thermal bremsstrahlung spectra: a high-energy one $(\mathrm{kT} \sim \mathrm{a}$ few $\mathrm{keV})$, and a low-energy one $(\mathrm{kT} \lesssim 1 \mathrm{keV})$. Apart from the pointlike emission of massive stars, this last component is diffuse, and covers the whole nebula. As shown by Montmerle and Dorland (1987), this last component may be entirely attributed to the unresolved emission from low-mass PMS stars having the same typical luminosity and surface density as observed in the nearby $\rho$ Oph cloud (Montmerle et al. 1983; see above, $\S$ 2.2.). At the distance of the nebula $(\approx 2.5 \mathrm{kpc})$, these stars are too faint to be optically visible individually, but the relevant ones would lie projected on the far side of the HII region, as is possible given the general orientation of the molecular cloud deduced from $\mathrm{CO}$ observations, which makes only a small angle to the line of sight. Once the high-energy tail of this component is subtracted ( $\approx 30 \%$ of the luminosity), the remaining X-ray luminosity is consistent with emission from shocks at the boundary of the inner cavity created by the massive stars present (and dominated by $\eta$ Car and the $3 \mathrm{~W}-\mathrm{R}$ stars associated with the nebula), according to the model of Dorland and Montmerle (1987). Essentially similar results may be obtained for other nebulae.

Hence, according to these ideas, there is a strong link between the presence of stellar winds inside giant HII regions and emission of X-rays at a temperature of several $\mathrm{keV}$. In turn, this suggests a possible link with the so-called "galactic [X-ray] ridge" (Montmerle 1986). This ridge, discovered by the Japanese satellite Tenma (Koyama 1987) and confirmed by EXOSAT (Warwick et al. 1985, 1988), is defined as the diffuse high-energy (several keV) emission seen to be associated with the galactic plane, once known localized sources (supernova remnants, compact binaries, etc.) have been removed. The angular resolution of the resulting composite "image", made up of pointings along different lines of sight, is very poor (a field of view of $\sim 1^{\circ}$ diameter for the EXOSAT $\mathrm{s} \mathrm{ME;} \mathrm{of} \sim 5^{\circ}$ for Tenma), but it is clear that the emission is thermal (systematic presence of a conspicuous heliumlike $\mathrm{Fe} 6.7 \mathrm{keV}$ line in the Tenma spectra) and corresponds to different temperatures along different lines of sight. The total luminosity is $L_{X}$, tot $\approx 1.2 \times 10^{38} \mathrm{erg} . \mathrm{s}^{-1}$.

\subsection{W-R STARS AND THE GALACTIC RIDGE}

Since many W-R stars lie inside giant HII regions, it is therefore tempting to suggest that, via their powerful winds, these stars make a significant contribution to the galactic ridge. Indeed, taking a typical mechanical luminosity $\mathrm{L}_{X, \mathrm{~W}-\mathrm{R}} \approx 4 \times 10^{34} \mathrm{erg} . \mathrm{s}^{-1}$ (see above), we can explain the observed luminosity with $\approx 3000$ such embedded $W-R$ stars. This figure is significantly higher than the estimated total number of W-R stars in the Galaxy (van der Hucht et al. 1988), but since many regions in the galactic plane are heavily obscured by 
dust, this number is in reality a lower limit: the actual number is unknown but could be compatible with the above figure. At any rate, the possibility of a connection between winds from massive stars (particularly the W-R stars) should be followed up when experiments with a better angular resolution at several $\mathrm{keV}$ (in particular the European satellite XMM, planned for the late 1990 's) become available.

\subsection{THE CONTRIBUTION OF SUPERNOVA REMNANTS}

Also efficient at producing X-rays in regions of massive star formation, and in particular in giant HII regions, are supernova remnants. However, if a supernova explosion occurs inside the HII region, the X-rays are efficiently produced only near the edge of the cavity, i.e., when the shock meets a dense medium while retaining a large velocity. Such a situation has been suggested to explain the high X-ray $(\sim \mathrm{keV})$ luminosities observed to be associated with "superbubbles" in the LMC , which reach 1037 erg.s $\mathrm{s}^{-1}$ (Chu and Mac Low 1990 and refs. therein). This interpretation is attractive, since such luminosities are much too high to be explained even by W- $R$ winds.

\section{W-R stars and nuclear $\gamma$-ray lines}

Recent observations by the SMM satellite and by several balloon experiments have revealed the existence of a line emission at $1.8 \mathrm{MeV}$ associated with the galactic plane, with a strong enhancement in the galactic center region. Owing to the very poor angular resolution of the instruments (typically more than $10^{\circ}$ ), the geometrical structure of the emission is still controversial. By contrast, the emission process is unambiguous, because the line is the signature of the $\beta^{+}$decay of ${ }^{26} \mathrm{Al}$. Since this radioactive isotope has a half-life of $\simeq 10^{6} \mathrm{yrs}$, the detection of the line means that "fresh" nucleosynthesis is going on; the total mass of ${ }^{26} \mathrm{Al}$ necessary to account for the $1.8 \mathrm{MeV}$ flux is $\sim 3 \mathrm{M}_{\odot}$, but this estimates depends somewhat on the assumed geometry of the emission (Prantzos and Cassé 1986, Prantzos, Cassé, and Arnould 1988).

The origin of the ${ }^{26} \mathrm{Al}$ itself is however unclear. Novae and SN do produce this isotope (see, e.g., Signore and Vedrenne 1988), but would produce too much ${ }^{27} \mathrm{Al}$ if they alone accounted for the observed ${ }^{26} \mathrm{Al}$. ON stars ("blue stragglers") have also been suggested, but, given the known number of such stars, they could account for only $\approx 10 \%$ of all the $26 \mathrm{Al}$ (Walter and Maeder 1989). W-R stars, on the other hand, could explain all the ${ }^{26} \mathrm{Al}$ only provided the galaxy contained some $10^{4}$ of them, which, even taking into account the uncertanties already mentioned above, seems too many (Prantzos, Cassé, and Arnould 1987). More likely, they may contribute to the ${ }^{26} \mathrm{Al}$ only a few times more than the $\mathrm{ON}$ stars.

However, in this context, an interesting connection can be made with the "galactic ridge", since the same stars could be responsible for at least a significant part of both the galactic ridge (via the interaction of their winds with the surrounding HII regions) and the diffuse nuclear $\gamma$-ray line emission (also via their winds, but this time spreading the ${ }^{26} \mathrm{Al}$ synthesized in their cores into the interstellar medium).

\section{High-energy $\gamma$-ray emission}

There may be links between giant HII regions and $\gamma$-ray emission even at higher energies. Following the early results by SAS-2, the COS-B satellite has mapped the galactic emission 
in the $\sim 30 \mathrm{MeV}-5 \mathrm{GeV}$ range, leading to the discovery of 25 "sources" (Bignami and Hermsen 1983). In reality, because of the poor angular resolution of these experiments (error box of $\sim 1^{\circ}$ radius), any localized region of diffuse emission within this error box can be proposed as the source counterpart. Molecular clouds, for instance, are good candidates since they represent concentrated gaseous masses immersed in the galactic cosmic ray flux (see above, $\$ 1.2$ ). On the other hand, mainly on the basis of coincidences along the line of sight, Montmerle (1979) had proposed the identification of $\sim 1 / 3$ to $1 / 2$ of the COS-B sources with giant HII regions containing SNRs; others, containing stars with strong stellar winds (especially W-R stars), were soon added to the list (see Montmerle 1985, 1988). The physical mechanism invoked is that in both cases strong shock waves are generated, which can accelerate protons in situ (by the so-called diffusive acceleration mechanism, see, e.g., Drury 1983, Völk 1988). Furthermore, the fact that the acceleration region is in both cases surrounded by large quantities of ionized gas $\left(\sim 10^{3}\right.$ to $\left.10^{5} \mathrm{M}_{\odot}\right)$ allows an efficient trapping by Alfvén waves of the accelerated particles, and consequently efficient collisions which produce high-energy $\gamma$-rays by pion-muon decay and bremsstrahlung. Using such a mechanism, and modelling giant HII regions like the Carina nebula and their associated molecular cloud in a simplified way, Montmerle and Cesarsky (1981), and Cesarsky and Montmerle (1983), have shown that a high-energy proton density of 10 to 100 times the average density in galactic cosmic rays should be present. Given the large shock energies available, to attain such a density requires an acceleration efficiency of only a few percent, hence should be easily attained. It will be the task of new experiments which a better angular resolution (like GRO, to be launched next fall) to test this model: one of the predictions is that the $\gamma$-ray emission of the HII region should be stronger than that of the neighbouring molecular cloud (itself already a strong $\gamma$-ray emitter because of the interaction of ambient galactic cosmic rays and its large mass). Giant HII regions containing W-R stars, like the Carina nebula, are therefore prime targets for these experiments.

\section{Summary and prospects}

Because they contain highly energetic objects which generate shock waves (stars with strong stellar winds, especially W-R stars, and/or supernova remnants), and/or release nucleosynthetic products (W-R stars), giant HII regions are important localized sources of high-energy emission, from $\sim \mathrm{keV} \mathrm{X-rays} \mathrm{to} \sim \mathrm{GeV} \gamma$-rays. They may be important even on a galactic scale (possible connection with the "galactic [X-ray] ridge" and the $1.8 \mathrm{MeV}$ line diffuse emission). The Table summarizes the various connections discussed in this paper.

More particularly in the context of W-R stars, it is also apparent from this Table that the study of high-energy emission from giant HII regions can tell us a lot about the external regions of these stars:

- X-rays tell us about the outer regions of the winds (as does the radio emission);

- nuclear $\gamma$-ray lines tell us about the nucleosynthetic yield of radioactive isotopes;

- high-energy $\gamma$-rays are related to the mass-loss energetics.

However, in the two last cases, the angular resolution is not sufficient to allow observations of single stars, so that for the time being we mostly have access to quantities integrated over geometrically relatively large regions. In the forthcoming years, several space experiments should help clarify the contribution of individual W-R stars to the highenergy emission of galactic plane, and it is hoped that, at the time of the next IAU Symposium on W-R stars, totally new results will be available in this field. 


\section{TABLE.}

\section{HIGH-ENERGY EMISSIONS FROM GIANT HII REGIONS}

\begin{tabular}{|c|c|c|c|c|c|c|}
\hline $\begin{array}{l}\text { Energy } \\
\text { domain }\end{array}$ & $\begin{array}{c}\text { Stars } \\
\text { (massive) }\end{array}$ & $\begin{array}{c}\text { Stars } \\
\text { (low-mass PMS) }\end{array}$ & $\begin{array}{l}\text { Wind } \\
\text { bubble }\end{array}$ & [SNR] & $\begin{array}{c}\text { W. shock } \\
\text { region }\end{array}$ & $\begin{array}{l}\text { HII } \\
\text { gas }\end{array}$ \\
\hline $\begin{array}{l}<\mathrm{keV} \\
\sim \mathrm{keV} \\
\mathrm{x} \mathrm{keV}\end{array}$ & + & + & + & + & $\begin{array}{l}+(1) \\
+\left({ }^{2}\right)\end{array}$ & \\
\hline $\begin{array}{l}\gamma \text {-ray lines } \\
(\mathrm{MeV}) \\
>30 \mathrm{MeV}-\mathrm{GeV}\end{array}$ & $\begin{array}{l}+\left({ }^{(3)}\right. \\
+\left({ }^{4}\right)\end{array}$ & & & & + & + \\
\hline $\begin{array}{l}\text { NT radio } \\
\mathrm{CR}: \mathrm{e}(>\mathrm{GeV}) \\
\mathrm{CR}: \mathrm{p}(>\mathrm{GeV})\end{array}$ & $\begin{array}{l}+ \\
+(6) \\
?(8)\end{array}$ & + & + & $\begin{array}{l}+ \\
+ \\
+\end{array}$ & $\begin{array}{l}?\left(^{5}\right) \\
+ \\
+\end{array}$ & $\begin{array}{l}+\left({ }^{7}\right) \\
+\left({ }^{9}\right)\end{array}$ \\
\hline
\end{tabular}

\footnotetext{
(1) Estimated from Breitschwerdt and Kahn (1988), see text, $\S 3$.

(2) In the model of Dorland and Montmerle (1987).

(3) W-R stars.

(4) W-R stars (Pollock 1987a)

(5) From the wind shock. Presumably buried in the thermal emission and/or absorbed by the ionized gas.

(6) Seen from the non-thermal radio emission.

(7) See Note (5).

(8) Possibly accelerated within the wind flow by shocks (turbulence, instabilites, etc.; see Cesarsky and Montmerle 1983).

(9) Indirectly visible via $\gamma$-ray emission.
} 


\section{REFERENCES}

Becker R.H., Boldt E.A., Holt S.S., Pravdo S.M., Rothschild R.E., Serlemitsos P.J., Swank J.H. (1976), Ap.J. (Letters) 209, L65.

Bertout C. (1989), Ann.Rev.Astr.Ap. 27, 351.

Bieging J.H., Abbott D.C., Churchwell E.B. (1989), Ap.J. 340, 518.

Bignami G.F., Hermsen W. (1983), Ann.Rev.Astr.Ap. 21, 67.

Bouvier J. (1990), Astr. Ap., in press.

Breitschwerdt D., Kahn F.D. (1988), M.N.R.A.S. 235, 1011.

Breitschwerdt D., Kahn F.D. (1989), M.N.R.A.S. 242, 209.

Campbell P.M. (1984), Phys. Rev. A 30, 365.

Cesarsky C.J., Montmerle T. (1983), Sp. Sci. Rev. 36, 173.

Chlebowski T. (1989), Ap.J. 342, 1091.

Chlebowski T., Harnden F.R., Jr., Sciortino S. (1989), Ap.J. 341, 427.

Chlebowski T., Seward F.D., Swank J., Szymkoviak A. (1984), Ap.J. 281, 665.

Chu Y.-H. (1983), Ap.J. 269, 202.

Chu Y.-H., Mac Low M.-M. (1990), Ap.J., in press.

Dorland H., Montmerle T. (1987), Astr. Ap. 177, 243.

Dorland H., Montmerle T., Doom C. (1986), Astr. Ap. 160, 1.

Drury L. O'C. (1983), Rep. Progr. Phys. 46, 973.

Feigelson E.D. (1987), in Proc. 5th Cambridge Cool Star Workshop, eds. J.L. Linsky and R. Stencel (Berlin: Springer), p. 455.

Gaetz T.J., Salpeter E.E. (1983), Ap.J.Suppl. 52, 155.

Hogg D.E. (1989), Astr.J. 98, 282.

van der Hucht K.A., Hidayat B., Admiranto A.G., Supelli K.R., Doom C. (1988), Astr. Ap. $199,217$.

Koyama K. (1987), Pub.A.S.J. 41, 665.

Koyama K., Asaoka I., Ushimaru N., Yamauchi S., Corbet R.H.D. (1990), Ap.J., in press.

Langer N. (1989), Astr. Ap. 220, 135.

Luciani J.F., Mora P., Pellat R. (1985), Phys. Fluids 28, 835.

Meaburn J., Solomos N., Laspias V., Goudis C. (1989), Astr. Ap. 225, 497.

Montmerle T. (1979), Ap.J. 231, 95.

Montmerle T. (1985), Proc. 19th Int. Cosmic Ray Conf., San Diego, 1, 209.

Montmerle T. (1986), Proc. Advances in Nuclear Astrophysics, Paris, eds. E. VangioniFlam et al. (Gif-sur-Yvette: Editions Frontières), p. 335.

Montmerle T. (1987), Proc. Starbursts and Galaxy Evolution, Les Arcs, eds. T.X. Thuan, T. Montmerle, and J. Tran Than Van (Gif-sur-Yvette: Editions Frontières), p. 47.

Montmerle T. (1988), Proc. NATO ASI Genesis and Propagation of Cosmic Rays, Erice, eds. M.M. Shapiro and J.P. Wefel (Dordrecht: Reidel), p. 131.

Montmerle T., André Ph. (1988), Proc. NATO ASI Formation and Evolution of Low-Mass stars, Viana do Castelo, eds. A.K. Dupree and M.T.V.T. Lago (Dordrecht: Reidel), p. 225.

Montmerle T., Cesarsky C.J. (1981), Proc. Int. School and Workshop Plasma Astrophysics, Varenna, ESA SP-161, 319.

Montmerle T., Koch-Miramond L., Falgarone E., Grindlay J. (1983), Ap.J. 269, 182.

Pollock A.M.T. (1987a), Astr. Ap. 171, 135. 
Pollock A.M.T. (1987b), Ap.J. 320, 283.

Prantzos N., Cassé M. (1986), Ap.J. 307, 324.

Prantzos N., Cassé M., Arnould M. (1988), Proc. 20th Int. Cosmic Ray Conf., Moscow, 1, 152.

Rosner R., Golub L., Vaiana G.S. (1985), Ann.Rev.Astr.Ap. 23, 413.

Seward F.D., Chlebowski T. (1982), Ap.J. 256, 530.

Signore M., Vedrenne G. (1988), Astr. Ap. 201, 379.

Völk H. (1988), Proc. 20th Int. Cosmic Ray Conf., Moscow, 7, 157.

Walter R., Maeder A. (1989), Astr. Ap. 218, 123.

Warwick R.S., Norton A.J., Turner M.J.L., Watson M.G., Willingale R. (1988), M.N.R.A.S. 232, 551.

Warwick R.S., Turner M.J.L., Watson M.G., Willingale R. (1985), Nature 317, 218.

Weaver R., McCray R., Castor J., Shapiro P., Moore R. (1977), Ap.J. 218, 377.

Williams P.M., van der Hucht K.A., Pollock A.M.T., Florkowski D.R., van der Woerd H., Wamsteker W.M. (1990), M.N.R.A.S. 243, 662.

\section{DISCUSSION}

Chu: The Rosette nebula's bubble is in the central cavity; the fat ring represents the ambient imperturbed medium. There does not seem to be disagreement between the nebula morphology and the energy conserving bubble model. Furthermore, the X-ray emission from Rosette is consistent with what the energy conserving bubble model predicts.

Montmerle: What you say can be true only if you forget about the age of the exciting stars. In the energy conserving model, the Rosette nebula should be very young, much younger than the age of the stars you find using mass losing stellar evolutionary models, i.e., several million years, even taking into account more recent work (with different prescriptions for overshooting, mass loss rates, etc.). Conversely, if you take the past evolution of the exciting $\mathrm{O}$ stars into account, the corresponding energy conserving bubble should now be much larger (radius of $30 p c$, as compared with the observed $\sim 10 p c$ ). An extensive discussion on this point is contained in Dorland et al. (1986); additional arguments are presented in Dorland and Montmerle (1987).

Walborn: I question the hypothesis that diffuse X-ray emission from giant $H I I$ regions is due to low-mass pre-MS stars. (1) The existence of such stars with a normal IMF in giant $H I I$ regions is an open question; (2) in Carina, Herrer and I found a very complex interstellar absorption-line velocity structure corresponding to soft X-ray energies, most likely originating in the massive stellar winds; (3) one of the three similar WN-A stars in Carina is embedded in dense surrounding material, and it only has very high velocity interstellar lines and strong X-ray emission, suggesting interaction between the wind and interstellar medium as the source of both.

Montmerle: First, the X-ray energies corresponding to the thermalization of very fast winds such as those of $\mathrm{O}$ stars or WR stars are typically of several $\mathrm{keV}$ (the exact energy depends on the assumed dissipation mechanism, see $\S 3$ of my paper). This is what has been found with OSO-8, the EINSTEIN MPC, and TENMA. This should not be confused with the sub-keV diffuse emission resolved by the "Einstein" IPC and reported by Chlebowski et al. As regards this last component, all I am saying is that, if you take the average soft $\mathrm{X}$-ray $(\sim \mathrm{keV})$ luminosity per $\mathrm{T}$ Tauri star, and assume they have the same surface density (within the outer layers of the clouds, i.e., those still transparent to the X-rays) as actually 
observed in nearby molecular clouds (e.g. Montmerle et al., 1983), and integrate over the emissive area, you find a very good agreement with the observed flux in Carina and Orion, after having removed the low-energy tail of the hard X-rays, if any. In this interpretation, the soft X-ray emission would not be diffuse, but simply unresolved. A detailed discussion in the case of the Carina nebula appears in Dorland and Montmerle (1987), and takes into account your work as available at the time. Now the real content in $T$ Tauri stars associated with giant $H I I$ regions is admittedly not known (they are too far away), but there is little doubt such stars must be present! And the results on the soft X-rays, in this interpretaion, merely imply that the formation rate of low-mass stars is about the same for all molecular clouds - a really interesting, but not too surprising, conclusion...

Jura: In the case of WR winds, you are looking to a non-standard composition with perhaps a lot of extra $C, H e$. What is the effect of that on their X-rays?

Montmerle: This is more a question for Pollock. To the extent that you are dealing with non-thermal X-rays, of course, then this depends only on the high energy electron content of the envelope. But, of course, if you have a different composition, and if you are, e.g., in a binary system, then the low energy part of the X-rays - if the X-rays are thermal - are probably affected by the presence of lines. But with EINSTEIN, there have been a few spectral data that point to this.

Pollock: X-ray observations are a fantastic way of finding out the chemical composition of the winds of hot stars, and soon there are going to be some observations from ROSAT which will tell us a lot about those things. I contend a difference between the mean X-ray luminosities of WN and WC stars, which tell you that they are chemically different to each other, and a number of other things as well. But, I also would like to make a couple of comments about what Walborn said about the WR stars in Carina. There are three WR stars there, one is much more luminous than the other two, it is fainter optically, it is hiding behind loads of absorbing material. But, I think that the evidence that big X-ray luminosity has something to do with the interaction with a dense interstellar medium is poor, because there is absolutely no evidence from the high angular resolution HRI observations that that $\mathrm{X}$-ray source is extended. It is unresolved in the HRI and you would expect to see some extension if the X-rays were coming from a bubble of some sort in the interaction with the interstellar medium. And as far as the high energy X-rays go from the Carina Nebula, with particular reference to the OSO-8 measurements, it is true to say that AG Car itself has a very hard X-ray spectrum. In the SSS, which was the good energy resolution instrument aboard EINSTEIN, AG Car has an almost flat, in fact slightly rising X-ray spectrum and I think that could account for the sort of OSO-8 picture you were showing there. So, I would just say, careful about that one!

Montmerle: I think this has been taken into account. Knowing the emission from AG Car itself and attributing also into this a kind of wind thermalization mechanism, this can be removed, and I was referring mostly to the X-ray emission without AG Car, even though the picture refers to everything. 


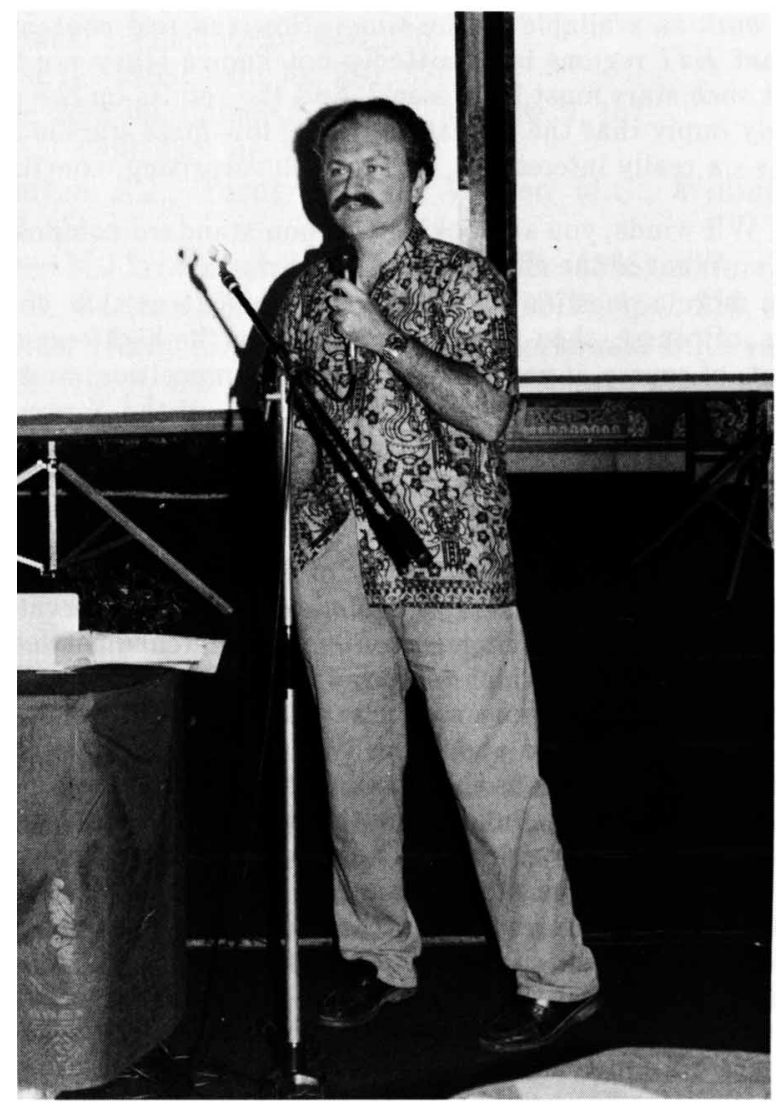

Thierry Montmerle 